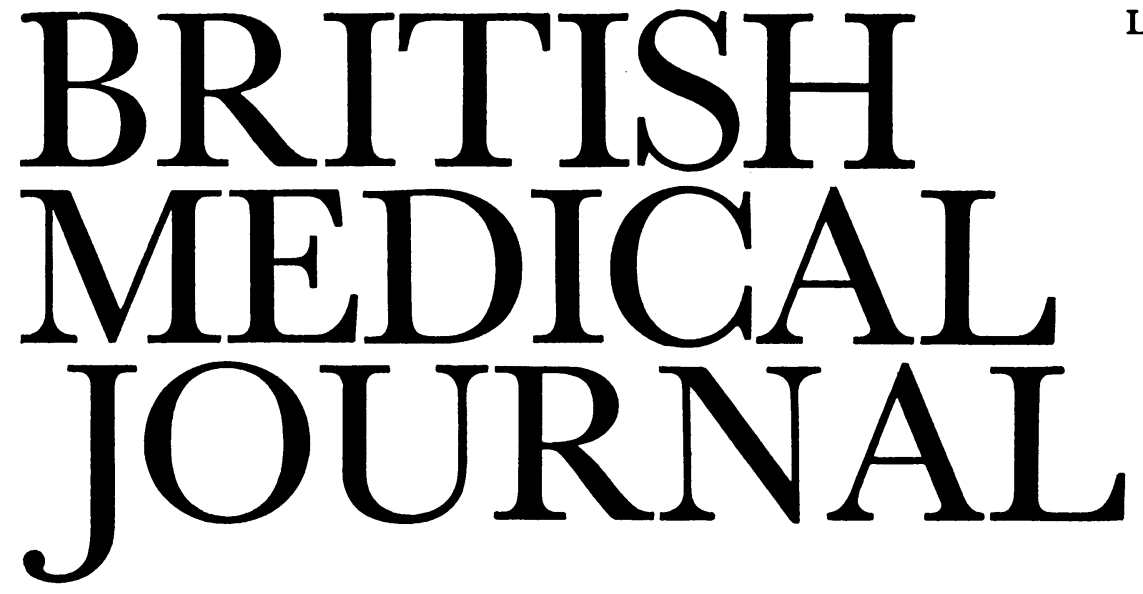

\title{
Acquired immunodeficiency syndrome, hepatitis, and haemophilia
}

People with haemophilia, their families, and their doctors feel threatened by the deluge of speculation about the possible side effects of treatment with blood products. Two topics hold their attention: the risk of contracting the acquired immunodeficiency syndrome (AIDS) and the risk of developing hepatitis and subsequent chronic liver disease.

Centers for Disease Control in Atlanta define a case of AIDS as "a reliably diagnosed disease that is at least moderately indicative of an underlying cellular immunodeficiency in a person who has had no known underlying cause of cellular immunodeficiency and no other cause of reduced resistance reported to be associated with that disease." For epidemiological purposes this definition is wide; it does not suggest a single aetiological agent, merely a similar response, or lack of it, to injury. It is important to recognise this in view of the difficulty in classifying the different disorders concerned and the current reaction of the media to announcements of "new" cases. Until diagnostic markers for the disorder are found terms like "confirmed AIDS" are unhelpful in defining what may well be a multifactorial disorder.

To date, patients fulfilling the Centers for Disease Control definition have included male homosexuals $(71 \%)$, intravenous drug abusers of both sexes $(17 \%)$, and people from Haiti $(5 \%)$. Heterosexual partners of people with AIDS or in a high risk group for the disorder $(1 \%)$, haemophiliacs $(1 \%)$, and recipients of blood products (other than haemophiliacs) with immunodeficiency $(1 \%)$ have also been reported. Some $4 \%$ of the 2259 cases notified in the United States and Puerto Rico by September 1983 fitted no particular pattern. ${ }^{1}$

Among the estimated 20000 people with haemophilia A (factor VIII deficiency) in the United States, 17 cases of AIDS have been reported. Ten patients have died, all with Pneumocystis carinii pneumonia. ${ }^{2}$ Two of the 17 cases had other risk factors for AIDS. No cases of Kaposi's sarcoma and haemophilia are known. Britain has 4592 people with haemophilia A known to the haemophilia centre directors, and two cases of haemophilia and AIDS have been reported to the Communicable Disease Surveillance Centre at Colindale. One patient with $P$ carinii pneumonia has died (G L Scott, personal communication), and the other has had opportunistic infections (A L Bloom, personal communication). Thus the reported incidence of AIDS in the two countries is about 0.8 per 1000 haemophiliacs. If all disorders of cellular immunity, thrombocytopenia, and lymphopenia are classed as cases of $\mathrm{AIDS}^{3}$ this figure may be an underestimate, but it does help to put the risks into perspective.

Liver function test results are abnormal in most severely affected haemophiliacs who have had repeated transfusions. The abnormalities are usually intermittent and are rarely associated with any clinical abnormality, though occasionally they may be associated with episodes of overt jaundice, be persistent, and be associated with chronic liver disease. Some patients have an enlarged liver or spleen or both on physical examination or on a scan of the liver and spleen. In a recent survey of 25 haemophiliacs having modest factor VIII replacement (18 000 units per patient a year) 24 had evidence of past exposure to hepatitis $\mathrm{B}, 18$ had abnormal liver function test results, 10 had an enlarged spleen, and eight had significant changes in the uptake of tracer by the liver. ${ }^{4}$ Thus despite an increase in the sensitivity of tests for hepatitis B virus and the screening of donors, this virus remains a hazard of blood transfusion, especially in the large pool concentrates prepared for haemophilia treatment. Most post-transfusion hepatitis is now, however, associated with non- $A$, non-B agents, presumably viral. As with the $B$ virus, non- $A$, non- $B$ hepatitis has been more prevalent in blood from paid rather than volunteer donors. ${ }^{5}$

So, in Britain where imported commercial concentrates accounted for $60 \%$ of the total factor VIII used in $1980,{ }^{6}$ what risk is there of serious harm from haemophilia treatment? Part of the answer is given by Fletcher and her colleagues (p 1754). In a study of 30 patients given a transfusion of factor VIII concentrate, four had evidence of unsuspected chronic liver disease, 17 developed hepatitis, and only two, who had received cryoprecipitate, remained unscathed. Twelve of 19 patients who received only National Health Service concentrate prepared from volunteer donor plasma, and all five patients who received a commercial concentrate, developed hepatitis. Although the results were influenced by previous transfusions, the striking finding was that hepatitis developed in all nine patients who had not previously received factor VIII concentrate. The authors suggest that the size of the pool of National Health Service concentrate ( $>3500$ donors a batch) has been increased to a point where the benefit conferred by the use of volunteer donor plasma might have been lost. But despite these gloomy figures the mortality from liver disease remains low, and only two British haemophiliacs died from hepatitis between 1974 and 1980 . Thus probably most of the 
observed changes in liver function represent chronic persistent rather than chronic active hepatitis. ${ }^{\text {? }}$

The commercial companies in the United States acted responsibly and quickly to exclude high risk donors, and similar action has now been taken in Britain, but in the absence of diagnostic serological markers putative agents of AIDS or of non-A, non-B hepatitis cannot be excluded from donor plasma. So what further measures can be taken to lessen the potential risks of AIDS and hepatitis? Studies in animals suggest that heat treatment might reduce transmission of non-A, non-B hepatitis, and manufacturers of factor VIII concentrates are poised to introduce heat treated preparations, although this may double the cost of treatment to the British market. It is too early to evaluate the effects of the new manufacturing processes, including the use of heat, which differ among companies, but protection is known not to be absolute. At least six patients under study have developed abnormal liver function test results after transfusion. There is no evidence that any product, commercial or volunteer, is free from the risk of transmitting AIDS. Patients with haemophilia B (factor IX deficiency) do not appear to develop AIDS despite multiple transfusions. This may simply be because haemophilia B is rare, but it might reflect a difference in the way factor IX concentrate is manufactured. Possibly what we are seeing among haemophiliacs is an entirely different disorder from that described in homosexuals-that is, one due to repeated antigenic challenge over many years rather than to a trans missible agent.

The median expectation of life in patients with severe haemophilia in Britain was estimated as near normal in $1980,{ }^{6}$ in contrast to 37 years in $1962 . .^{\circ}$ This increase in longevity and the improved quality of life are due entirely to the widespread introduction of factor VIII concentrates and to comprehensive care. When AIDS was first linked with haemophilia, and the extent of the problem in the United States was unknown, some centres curtailed planned surgery and home treatment. Nevertheless, most have now reverted to their routine programmes, and throughout the world the opinion of the majority is that the risk of haemorrhage and its complications far outweighs the risk of developing AIDS or chronic liver disease.

For the moment, however, it seems sensible to treat very young severely affected children with cryoprecipitate rather than concentrates. Alternative methods of raising factor VIII activity with desmopressin (DDAVP), ${ }^{9}$ danazol, ${ }^{10}$ or perhaps the new porcine material should be used in mildly affected haemophiliacs, people with von Willebrand's disease, and carriers of these disorders. There may be a case for long term prophylaxis with co-trimoxazole in those haemophiliacs presenting with lymphopenia and unexplained fever or wasting because all the deaths to date have been associated with infection with $P$ carinii. Prophylaxis against this organism is effective, ${ }^{11}$ and its suppression may buy time for the patient with AIDS. There is no evidence of casual transmission of AIDS to health care staff, although the Americans recommend the adoption of similar precautions to those already in use for hepatitis B when handling specimens from suspected cases. ${ }^{12}$ The most important precaution of all, however, is to maintain a high level of surveillance of the haemophilic population.

Peter Jones

Director,

Newcastle Haemophilia Centre,

Royal Victoria Infirmary,

Newcastle upon Tyne NE1 4LP
${ }^{1}$ Centers for Disease Control. Acquired immunodeficiency syndrome (AIDS) update-United States. MMWR 1983;32:465-7.

${ }^{2}$ Marwick C. 'Contaminated' plasma: no automatic recall. fAMA 1983; 250:1126-7.

${ }^{3}$ Curran JW. AIDS-two years later. $N$ Engl f Med 1983;309:609-10.

${ }^{4}$ Meyer WH, Levin J, Ness PM, et al. Abnormalities of the spleen and liver in patients with hemophilia. Am f Hematol 1983;14:235-46.

${ }^{5}$ Seeff LB. Post transfusion hepatitis in haemophilia. In: Seligsohn U, Horoszowski H, eds. Haemophilia. Chichester: John Wiley and Sons Ltd, $1981: 131-9$.

${ }^{6}$ Rizza CR, Spooner RJD. Treatment of haemophilia and related disorders in Britain and Northern Ireland during 1976-80: report on behalf of the directors of haemophilia centres in the United Kingdom. Br Med $\mathcal{F}$ $1983 ; 286: 929-33$.

7 White GC II, Zeitler KD, Lessesne HR, et al. Chronic hepatitis in patients with hemophilia A: histologic studies in patients with intermittently abnormal liver function tests. Blood $1982 ; 60: 1259-62$

${ }^{8}$ Biggs $\mathrm{R}$, ed. The treatment of haemophilia $A$ and $B$ and von Willebrand's disease. Oxford: Blackwell Scientific Publications, 1978:25.

${ }^{9}$ Mannucci PM, Ruggeri ZM, Pareti FI, Capitanio A. 1-Deamino-8-Darginine vasopressin: a new pharmacological approach to the management of haemophilia and von Willebrand's disease. Lancet 1977; : 869-72.

${ }^{10}$ Gralnick HR, Rick ME. Danazol increases factor VIII and factor IX in classic hemophilia and Christmas disease. N Engl $f$ Med 1983;308: 1393-5.

${ }^{11}$ Hughes WT, Kuhn S, Chaudhary S, et al. Successful chemoprophylaxis for Pneumocystis carinii pneumonitis. N Engl f Med 1977;297:1419-26.

${ }^{12}$ Centers for Disease Control. Acquired immunodeficiency syndrome (AIDS): precautions for health-care workers and allied professionals. $M M W R \quad 1983 ; 32: 450-1$.

\section{Assessment of pituitary function}

Testing pituitary glandular function is only part of the assessment of pituitary disease, for it does not assess damage to the optic pathways or define an empty sella. Furthermore, it usually fails to measure the rate of secretion of the pituitary hormones, though their metabolic clearance rate may be studied. ${ }^{1}$

Measurement of plasma concentrations of hormones does give a better indication of pituitary function than clinical observation-sometimes because we lack knowledge of their function (for example, growth hormone in the adult); because an intermediate substance is concerned (for example, somatomedin with its action on skin and skeleton $)^{2}$; or because of the variability of the hormones' tissue receptors.

Most hormones are measured by radioimmunoassay, for cytochemical assays, despite their promise of greater sensitivity, are difficult to carry out except in a few specialised laboratories. $^{3}$ Since, however, the part of a hormone that reacts antigenically with a particular antibody is not always strictly, or even strongly, linked with the hormone's tissue reactive group, particularly when a neoplasm secretes the hormone or its homologue, ${ }^{4}$ the cruder bioassays remain useful as reference standards. Indeed, this explains why many prefer to express the results of a hormone assay in units of measured activity, even if these are not entirely comparable because of the different antibodies used.

Hormone concentrations may be measured in a random sample or in specified conditions (when a steady state might be expected), or after deliberate manipulation-classically by stimulation to test deficiency and by suppression when overactivity is suspected. Random measurement is acceptable for thyroid stimulating hormone if only because the widely used radioimmunoassay is too insensitive to separate abnormally low from healthy concentrations. ${ }^{5}$ With prolactin care must be taken to avoid disturbing circumstances, for it is one of several 